\title{
A Study on Construction Method of Consensus Measure Space of Macro-seismic Anomalies
}

\author{
Weidan Wang ${ }^{1,2}$, Chongfu Huang ${ }^{1,2,3, *}$ \\ ${ }^{l}$ State Key Laboratory of Earth Surface Processes and Resources Ecology, Beijing Normal University, \\ Beijing 100875, China \\ ${ }^{2}$ Academy of Disaster Reduction and Emergency Management, Faculty of Geographical Science, Beijing Normal \\ University, Beijing 100875, China \\ ${ }^{3}$ Beijing Cazl Technology Service Co., Ltd., Beijing 100088, China
}

Received 28 June 2017

Accepted 10 July 2017

Abstract

\begin{abstract}
Macro-anomalies are so complex that it is difficult to carry out the systematic quantitative analysis macro-anomalies because it is lack of measure space. It's more difficult to find the relationship between micro-anomalies and earthquakes. In the paper, the theory system of macro-anomalies group's measure space which is expressed by the fuzzy relationship matrix is set up. The concept of macro-anomalies group is put forward. Scene, elements and attributes are used to describe the relationship among macro-anomalies group, macro-anomalies and basic information of macro-anomalies. Based on the expression of basic information and the evaluation information, according to properties of the elements of a scene, basic indicators that describe macro-anomalies group can be calculated. The generation of results based on consensus of the first-line earthquake workers' evaluation of macro-anomalies group's comprehensive abnormal degree is expounded. The specific process and related algorithm is raised that use information diffusion technique calculate the fuzzy relationship matrix to express macro-anomalies group's measure space, and it takes basic indicators of macro-anomalies group as input, the evaluation information of first-line earthquake workers as output.
\end{abstract}

Keywords: Earthquake, Macro-anomaly, Measure space, Information diffusion, Internet of intelligences.

\section{共识性的地震宏观异常群测度空间及其构建方法研究}

\section{王蔚丹 ${ }^{1,2}$, 黄崇福 $1,2,3, *$}

1. 北京师范大学地表过程与资源生态国家重点实验室，北京 100875

2. 北京师范大学地理科学学部减灾与应急管理研究院, 北京 100875 , 中国 3. 北京崇安智联科技服务有限责任公司，北京 100088 ，中国

摘＼cjkstart要:针对人们缺少综合度量地震宏观异常的测度空间，难以开展系统性的量化分析，更难以寻找宏观异 常与地震之间可能蕴含的关系等问题, 本文建立了用模糊关系矩阵表达地震宏观异常群测度空间的理论体系: 提 出了宏观异常群的概念，利用情景、要素、属性来描述宏观异常群、宏观异常和宏观异常基本信息之间的关系; 进行了宏观异常群基本信息及评判信息的表达，根据构成情景的要素的基本属性一宏观异常基本信息，可以得到 描述情景一宏观异常群的基本指标; 阐述了一线地震工作者对宏观异常群情景的综合异常程度进行评判得到共识

\footnotetext{
* Corresponding author: E-mail: hchongfu@bnu.edu.cn. Post address: Academy of Disaster Reduction and Emergency Management, Beijing Normal University, No.19 Xinjiekouwai Street, Beijing 100875, China

This project was supported by the National Natural Science Foundation of China (41671502), and partly supported by the Beijing Cazl Technology Service Co., Ltd..
} 
性结果的过程; 将宏观异常群的基本指标作为输入, 一线地震工作者对宏观异常群情景整体异常程度的共识性结 果作为输出, 提出了用信息扩散技术计算模糊关系矩阵表达地震宏观异常群测度空间的具体过程和相关算法。

关键词: 地震, 宏观异常, 测度空间, 信息扩散, 智联网

\section{1. 引言}

现阶段对宏观异常的统计分析缺乏大量可靠、完 备的数据, 导致宏观异常的研究裹足不前。现阶段利 用统计方法对宏观异常现象进行分析, 很难再有突破 性进展, 只能不断积累数据。宏观异常种类繁多, 表 现形式多样, 仅已知的动物异常就有几十种之多, 各 个异常又有具体的表现形态。在缺乏大量可靠、完备 宏观异常数据, 进行统计分析难以得到行之有效规律 的情况下, 拿到宏观异常信息时, 该如何分析处理这 些信息成为一个问题。

目前的宏观异常研究缺乏系统化的量化工具。宏 观异常的认识和运用主要由地震专家来完成, 无论是 宏观异常的现场核查, 还是对宏观异常进行宏观把握, 辅助地震预报, 都离不开专家的参与。我们把专家看 作一个个高级智能体, 基于智联网平台 ${ }^{[1]}$, 利用一线 地震工作者的经验和知识, 建立一个共识性的测度空 间综合度量宏观异常, 以便开展系统性的科学研究。

\section{2. 宏观异常群相关概念}

宏观异常: 指人类用感官可直接觉察到的自然界 中的不正常现象 ${ }^{[2]}$ 。

大震前一般是有宏观异常的。我国近四千年的文 化历史资料中有丰富的关于宏观异常资料的记载。将 历史资料中出现频率较高的宏观异常, 如井水浑浊, 翻花冒泡, 断水自流; 蛇冬眠时出洞, 老鼠惊慌乱跑, 狗成群乱咬乱叫, 大猫叺着小猫搬家等, 称为典型异 常。宏观异常一般不会孤立出现, 宏观异常的 “宏” 不仅体现在人类用感官就可以察觉到, 还表现为它一 般成群地出现在一定的空间范围内。

宏观异常表现形式多样, 种类多达几百种, 异常 现象的表现更是数以千计。宏观异常如此复杂, 其是 否出现, 又有很大的不确定性。相对单个的宏观异常 而言, 大规模的群体性的宏观异常, 对辅助判断地震 是否有可能发生, 更有指示意义。由此我们提出宏观 异常群的概念。

宏观异常群: 指在一定时空范围发生的多个宏观
异常组成的群体。

不同地震宏观异常现象存在地域上的相关性和时 间上的同步性特征，历史资料及文献中对于比较显著 的群发性宏观异常, 一般用种类多、数量多、范围广、 规模大、随时间剧烈变化等来描述。由于缺少综合量 化的理论和方法, 加之人们还不能在震前及时汇集和 处理宏观异常群信息, 宏观异常对地震预报的辅助决 策尚待改进。

我们把一个个的宏观异常看成一个个的异常要 素, 由若干个宏观异常构成了宏观异常群, 也就在某 个时间点上形成了关于一个地域的异常情景。例如, 7 个宏观异常组成了一个宏观异常情景, 投影在平面上 用地图显示就形成了图 1 中所示。为了综合度量某一 时空范围出现的宏观异常, 提出宏观异常群严重度的 概念。

宏观异常群严重度：指宏观异常群的综合异常程 度。

宏观异常群严重度类似于地震灾害中建筑物的破 坏严重度程度, 是一个综合的量。一个宏观异常群的 异常程度, 由所出现的宏观异常的种类、数量、范围、 规模以及单个宏观异常的异常幅度等指标所决定。用 区间 $[0,10]$ 中一个适当的数值来表示严重程度。 0 为无 异常; 10 为非常严重。

本文的主要工作, 就是探讨如何根据宏观异常群 中的异常种类、数量、范围、规模以及单个宏观异常 的异常幅度等, 计算宏观异常群严重度, 从而综合度 量宏观异常, 为辅助地震预测提供信息。

\section{3. 宏观异常群信息的表达}

\section{1 宏观异常群信息的表达}

我们把一个宏观异常群看成一个情景, 它由若干 个要素——宏观异常组成。每个宏观异常都有它自身 的属性, 如宏观异常所属类别、发生时间、持续时间、 发生地点 (经纬度)、异常描述及异常所处环境等。图 2 展示了宏观异常群层次结构示意图。

一个宏观异常群记为 $G$, 它由多个宏观异常 $M_{1}$, 


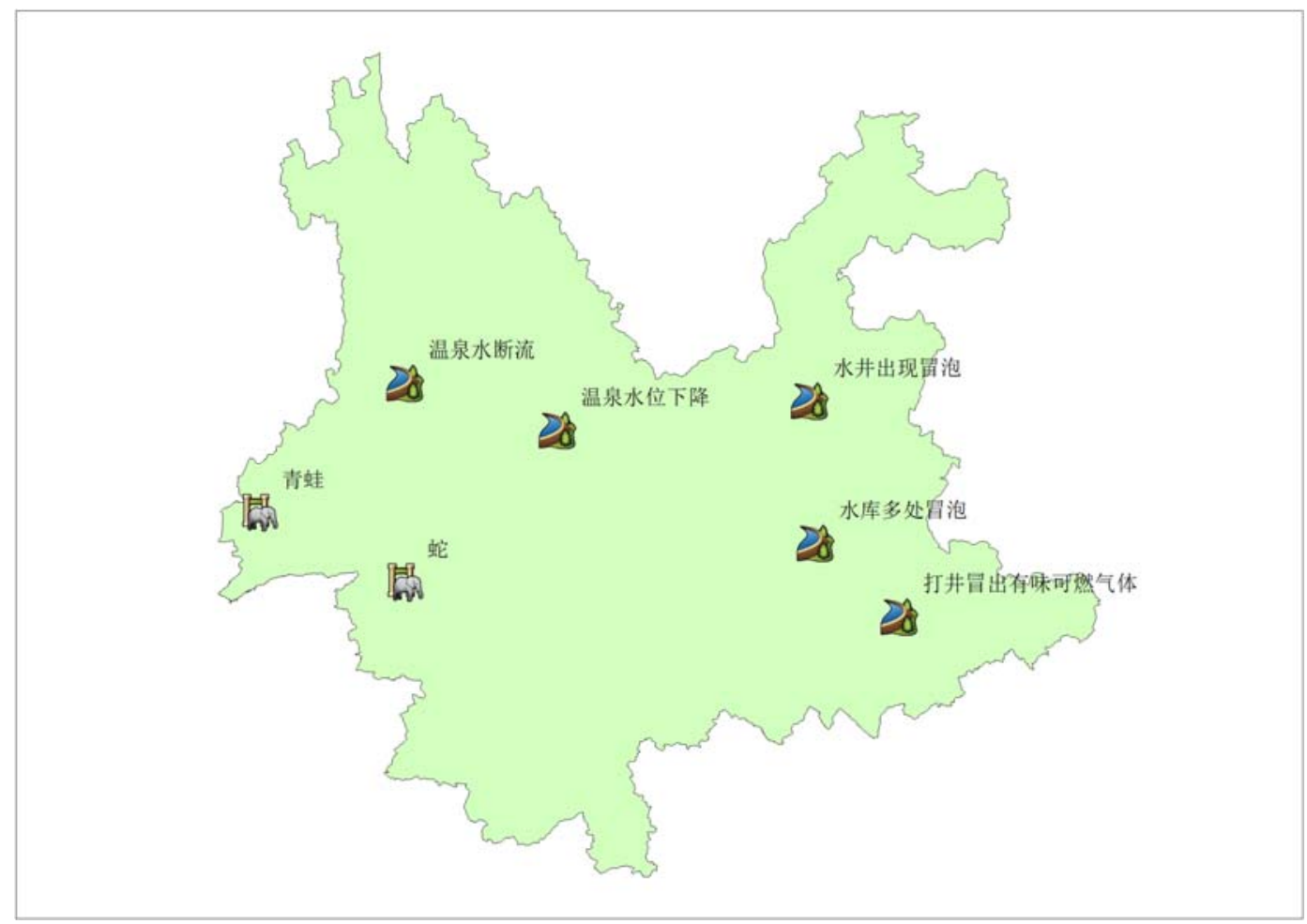

图 1. 宏观异常群情景

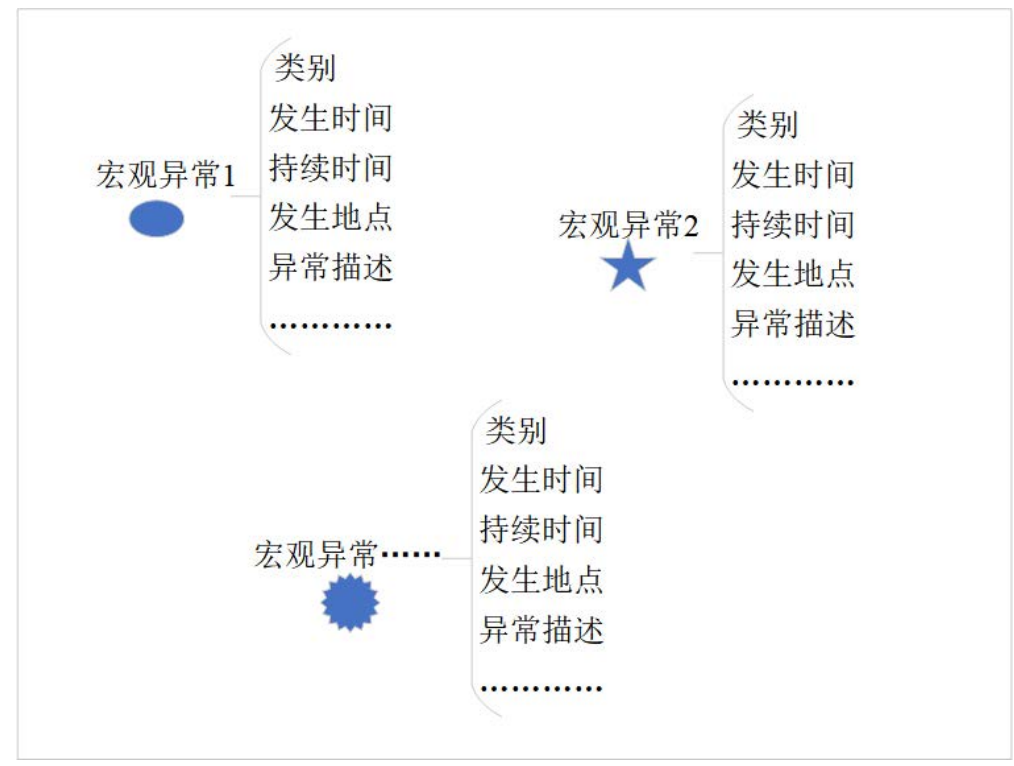

图 2. 宏观异常群层次结构示意图

$M_{2}, \ldots$ 构成。一个宏观异常由多个属性 $f_{1}, f_{2}, \ldots$ 进行描 述。一个宏观异常所属类别、发生时间、持续时间、 发生地点 (经纬度)、异常描述及异常所处环境等, 均 是其属性。式 (1) 表达了由 $n$ 个宏观异常构成的一个
宏观异常群 $G$ 。式 (2) 表达了由 $I$ 个属性描述的一个 宏观异常 $M$ 。

$$
\begin{gathered}
G=\left(M_{1}, M_{2}, \ldots, M_{n}\right) \\
M=\left(f_{1}, f_{2}, \ldots, f_{l}\right)
\end{gathered}
$$


假设宏观异常群 $G$ 由 $n$ 个基本单元一一宏观异常 来组成, 如式 (1) 所示, $M_{j}$ 表示构成宏观异常群的第 $j$ 个宏观异常, $j=1,2, \ldots, n$ 。根据宏观异常历史资料, 一 条宏观异常信息通常包括异常的类别、种类、出现时 间、结束时间、出现地点以及宏观异常现象的详细描 述等。若宏观异常 $M_{j}$ 可以通过 $l$ 个属性特征来表示, 则 $M_{j}$ 的属性特征集 $f_{j}$ 可具体地表示为式 (3):

$$
f_{j}=\left\{\left(i d_{j}, v_{j}, t_{j}, x_{j}, y_{j}, d_{j}, a_{j}\right) \mid j=1,2, \cdots, n\right\}
$$

$i d$ 是异常的唯一标识, $v_{j}$ 表示宏观异常群 $G$ 的第 $j$ 个 宏观异常 $M_{j}$ 所属的异常类别, $t$ 是异常发生的时间, 异常发生的地点这里用 $x, y$ 表示, $d$ 是对异常现象的具 体表述, $a$ 表示异常幅度, 即该异常区别于正常的显 著程度。

为了尽可能地将所有的异常都包括进来, 参照已 有的宏观异常历史资料, 我们采用《地震宏观异常预 报方法》一书的分类体系, 许敦煌对汶川地震前宏观 异常进行现场调查 ${ }^{[3]}$, 张小涛等对汶川地震前后出现

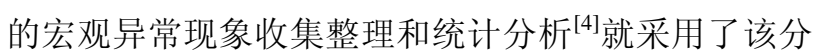
类方法, 上面所述的气象异常有中期异常 (旱涝异常) 和短期异常 (气温、地温、湿度、气压、降雨、风等 气象变化异常), 本文将第五类宏观异常表述为气候气 象异常, 具体分类情况见文献 ${ }^{[5]}$ 。

$V$ 是属性异常类别对应的属性特征值的集合, 前 文我们把宏观异常分为五大类, 可用式 (4) 来表示:

$V=\left\{V_{1}, V_{2}, \ldots, V_{5}\right\}$

$=\{$ 生物异常, 地下流体异常, 地球物理场异常, 地质现象异常, 气候气象异常\}

以往研究地震和宏观异常的关系时, 我们常常统 计震前出现的宏观异常种类、数量, 时空分布规律等, 较少将宏观异常本身反应的强烈、明显程度加以考虑。 我们把宏观异常反应的强烈、明显程度称为异常幅度。 异常幅度也是描述宏观异常的一个重要指标。

地震宏观异常涉及到异常种类繁多, 异常表现形 式多样, 很难有一个统一的标准。异常幅度 $a$ 带有很大 的主观性, 可以根据宏观异常具体表现形态加以判断, 或者从异常描述判断其异常幅度。为了研究方便, 可 将异常幅度按照级别加以划分, 如在描述地下水和地 下油气时可能出现水位大幅度上升; 水位大幅度下降; 枯井开始冒水; 水位不断升高, 同时伴有响声; 泉水 流量剧增; 泉水流量减少或断流; 井水 (泉水、水库、
龙洞等）发浑; 水变色、翻花冒泡、变味或有异味; 飘油、打旋、有响声; 田里冒水或喷沙; 水温升高或 降低等词句。李坤、李秀英在研究地下水和强震之间 的关系时, 将异常分为一般异常和重要明显异常两种。 一般异常, 主要表现为水位上升、下降; 冒泡、水变 浑等。重要明显异常, 主要以如井水突然自流、地面 翻砂冒水、水位忽升忽降和水变色等形式出现, 他们 提出后者对发震时间和震中预报有极其重要的作用 [6]。根据他们的区分方式, 为了我们的研究方便, 这 里将异常幅度分为两类, 一般异常和重要明显异常。 异常幅度 $a$ 对应的属性特征值的集合可具体地表示为 式 (5):

$$
A=\left\{A_{1}, A_{2}\right\}=\{\text { 一般异常, 重要明显异常 }\}
$$

具体地, 对地下流体异常来说, 将变色、自流、 井喷、翻砂冒水、水位忽升忽降作为重要明显异常, 水位上升、下降、流量改变、翻花冒泡、水变浑等作 为一般异常。对生物异常来说, 区域内群体性异常、 或者反应特别剧烈等可作为重要明显异常, 如 2000 年 9 月 12 日青海兴海 6.6 级地震前, 在 “震中区温泉一 带 95\%以上狗有异常反映, 狗异常狂犬, 且声音凄凉, 拖声很长, 似狼瞣声” 将其看作重要明显异常; 1997 年 5 月 31 日福建永安地震前 “胶南县百灵鸟笼之中乱 飞乱跳致死” 也可作重要明显异常。其它的可看作一 般异常。地声、地磁、地光及有感地动等地球物理场 异常以及地质现象和气象气候异常难以判别其异常幅 度, 暂时将其看作一般异常。

\section{2 宏观异常群的描述指标}

以往对于比较显著的群发性宏观异常, 一般用种 类多、数量多、范围广、规模大、随时间剧烈变化等 来描述。这些都是对出现在一定时空范围内根据式 （3）对某个宏观异常群中的单个宏观异常的异常类 别、发生时间、发生地点等属性信息进行统计, 可得 到该群出现的宏观异常个数, 出现的异常种类数, 时 间、空间分布情况等。除此之外, 群体的综合异常程 度还和个体的异常幅度有关。由此, 我们用种类数量、 分布情况和个体强度这些参量来描述宏观异常群, 具 体地, 种类数量指宏观异常出现种类数和异常总数, 即出现了哪几类, 共多少起异常; 分布情况主要用空 间密集度和时间频度来表示; 个体强度主要是指一起 
宏观异常表现的强烈程度。其中, 空间密集度指宏观 异常总量在空间范围的分布密度, 时间频度指宏观异 常在单位时间内出现的次数。

不同异常现象在空间上的相关性和时间上的同步 性是在宏观异常研究中尤其要关注的内容, 在本文中, 为了简化研究, 遵循从简单到复杂的原则, 采用种类、 总量、空间密集度、异常幅度来建立相应的测度空间。 假定宏观异常群分布在一定的时间范围内, 时空演化 的整体研究可以在后续研究中来实现。

根据前面的分析，把宏观异常划分为 5 大类， $N$ 表示宏观异常群中各类异常出现的次数集合, 用式 (6) 表示。

$$
N=\left(N_{1}, N_{2}, \cdots, N_{5}\right)
$$

$N_{1}$ 表示宏观异常群中第一类异常出现的个数, $N_{j}$ 表示 宏观异常群中第 $j$ 类异常出现的个数, 其中, $j=1,2, \ldots, 5$ 。这样, 该宏观异常群出现的异常类别数 及异常总起数就能计算出来。

空间密集度即宏观异常群在空间上密集程度。可 以用单位面积内的宏观异常总量来表示, 即总起数/面 积。例如, 在一定时间范围内出现三起异常, 分别分 布在西北、东南和东北, 可知其分布及其稀疏, 密集 程度低。相对来说, 如果在云南省内东北部出现了三 起宏观异常, 其在空间上的分布密集程度较高。 $\left(x_{j}, y_{j}\right)$ 表示宏观异常群中第 $j$ 起异常的位置, 据此可计算出 宏观异常分布范围, 用其覆盖面积来表示, 利用宏观 异常群出现的异常总量和面积可得到空间密集度指标 $C$ 。如此, 只要将宏观异常群的面积表现出来即可。 接下来看一下宏观异常群情景的形式化表达。

\section{3 宏观异常群情景及其评价信息的表达}

宏观异常群情景的表达。一个宏观异常群可以看 作一个宏观异常情景, 不失一般性, 设有 $\kappa$ 个宏观异 常情景，则

$$
S=\left\{S_{1}, S_{2}, \cdots, S_{\kappa}\right\}
$$

其中, $S_{i}$ 表示第 $i$ 个宏观异常情景, 其中 $i=1,2, \ldots, \kappa$ 。

假设第 $i$ 个情景 $S_{i}$ 由 $n$ 个基本单元组成, 则 $S_{i}$ 可 表示为式 (8):

$$
S_{i}=\left(F_{1}, F_{2}, \cdots, F_{n}\right)
$$

其中, $F_{i j}$ 表示构成第 $i$ 个情景的第 $j$ 个宏观异常要素, 其中 $i=1,2, \ldots, \kappa ; j=1,2, \ldots, n$ 。若宏观异常要素 $F_{i j}$ 可以
通过 $l$ 个属性特征来表示, 则 $F_{i j}$ 的属性特征集 $f_{i j}$ 可表 示为式 (9):

$$
f_{i j}=\left\{f_{i j 1}, f_{i j 2}, \cdots f_{i j l}\right\}
$$

其中, $f_{i j b}$ 表示构成第 $i$ 场情景的第 $j$ 个要素所具有的第 $b$ 个属性特征。

为了后续计算方便, 我们对宏观异常群构成的情 景信息进行综合表达。第 $i$ 场情景可用式（10）来表 示。

$S_{i}=\left\{\left(i d_{i}, N T_{i}, N V_{i}, N_{i 1}, N_{i 2}, \cdots, N_{i, 5}, \gamma_{i}\right) \mid i=1,2, \cdots, \kappa\right\} \quad(10)$

其中, $S_{i}$ 表示第 $i$ 个情景, id 是情景的唯一标识, $N T$ 表示情景 $S$ 包含的要素总数, 即宏观异常个数, $N V$ 是情景中异常类别数, $N_{i 1}$ 表示第一类异常 $V_{1}$ 在情景 $S_{i}$ 中出现的个数, $N_{i, 2}, \cdots, N_{i, 5}$ 以此类推。 $\gamma_{i}$ 表示情景 $i$ 中宏观异常覆盖的面积。

宏观异常情景评价信息的表达。假设呈现出一个 宏观异常群情景, 一线地震工作者对该情景的综合异 常程度进行评判, 这个评判信息怎么表达呢? 目前地 震的相关研究大多还处于初级阶段, 特别是宏观异常 在实际工作中发挥作用, 更多的是依靠经验。我们要 建立相关的智联网服务平台, 一线地震工作者们可根 据自身的经验和智慧对平台推送的宏观异常群情景进 行整体把控, 得出一个判断结论。

可以直接把自然语言映射成某个数值或数值区 间。例如把严重程度从不严重到非常严重映射到 $[0,10]$ 区间上, “比较小，不太严重” 转换为数值 3。有时为 了方便, 我们常常分区间来表达, 例如可将 $[0,10]$ 划分 为 5 个级别, [0-2]表示不严重, [2-4]表示不太严重, [4-6]表示严重, [6-8]表示比较严重, [8-10]表示非常严 重。直接选择和判断最接近的区间, 这个过程其实是 进行逻辑匹配的过程。不论是直接映射成数值还是直 接选择区间，都不可避免地丢失了很多信息。把这些 描述转换成某个论域上的模糊子集可以更准确地表达 模糊评价信息。我们要做的是将这种模糊判断通过画 图画出来。

如图 2 所示, 对出现的宏观异常组成的群体强度 做一个整体评判。横轴表示针对某一宏观异常群中出 现的全部宏观异常的种类、数量、范围、规模以及单 


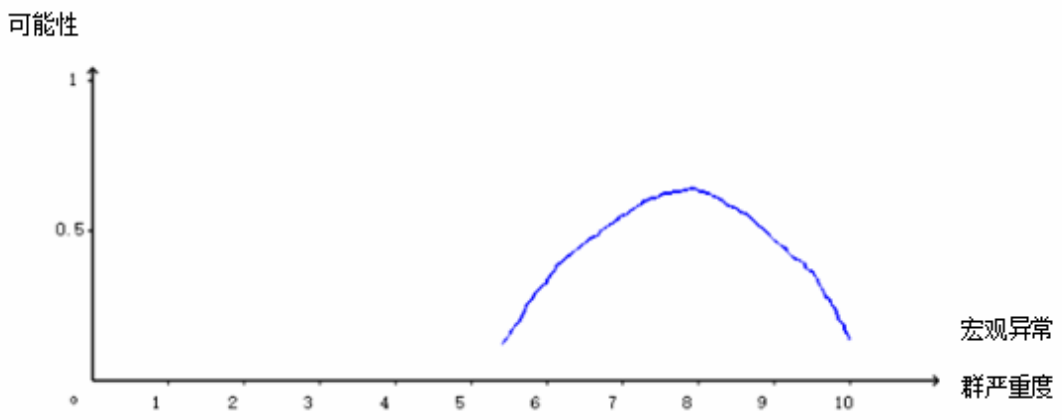

图 3. 一线地震工作者将评判结果进行模糊输入

个宏观异常的异常幅度等做出的严重程度判断, 数值 越大表示越严重, 用区间 $[0,10]$ 中一个适当的数值来表 示, 这个数值就是前面提到的宏观异常群严重度。这 里不严重为 0 , 非常严重为 10 。纵轴表示对相对应严 重程度的可能性判断, 可以理解为信度或者隶属度, 是对相对宏观异常群严重度的可能性判断。

一线地震工作者对群体异常严重程度的评判是模 糊的, 前面提到了宏观异常群严重度和隶属度的概念, 我们以 $\mathrm{Z}$ 来表示宏观异常严重度。出现一个宏观异常 群, 一线地震工作者通过画曲线的形式模糊输入进行 评判, 这个判断是连续的, 存储的时候就需要根据需 要进行采样, 得到离散化的一组数据。这样每次评判 都可以得到一组 $(v, p)$ 数据, 其中 $v$ 可看做宏观异常群 严重度 $\mathrm{z}$ 的监控点空间, $p$ 是对应的隶属度。

假设一共进行了 $k$ 次评价, 记 $\theta=\{1,2, \cdots, \beta\}$, 取 宏观异常群严重度的离散论域

$v=\left\{v_{1}, \quad v_{2}, \cdots, v_{\beta}\right\}, v_{j} \in[0,10]$,

第 $i$ 次评价结果记为 $J_{i}$, 则

$$
J_{i}=\left\{\left(v_{i 1}, p_{i 1}\right),\left(v_{i 2}, p_{i 2}\right), \cdots,\left(v_{i \beta}, p_{i \beta}\right)\right\}
$$

$p_{i j}$ 表示 $v_{i j}$ 对应的隶属度, 其中 $i=1,2, \ldots, k ; j=1,2, \ldots, \beta$ 。 那么

$$
p_{i}=\left\{p_{i 1}, p_{i 2}, \cdots, p_{i \beta}\right\}
$$

式（12）表示组成离散论域的这些标准点属于第 $i$ 次评价信息这个模糊子集的隶属度集合。

用 $p_{i, \text { maxi }}$ 表示第 $i$ 次评价中最大隶属度, 则 $\exists \operatorname{maxi} \in \theta$ ，使得

$$
p_{i, \operatorname{maxi}}=\max \left(p_{i}\right)
$$

其中, $i=1,2, \cdots, k$ 。

$v_{i, m p}$ 表示最大隶属度对应的宏观异常群严重度, 称其为最可能宏观异常群严重度。

$$
v_{i, m p}=v_{i, \operatorname{maxi}}
$$

其中, $i=1,2, \cdots, k$ 。同样 $m p \in \theta$ 。

例如，假设图 3 所示是第 3 次评价所绘制的模糊 输入曲线, 令

$$
v=\left\{v_{1}, v_{2}, \cdots, v_{m}\right\}=\{0,1,2, \cdots, 10\}
$$

根据所绘制的曲线, 评价结果可用一组数值表示

$$
\begin{aligned}
J_{3}= & \left\{\left(v_{3,1}, p_{3,1}\right),\left(v_{3,2}, p_{3,2}\right), \cdots,\left(v_{3,11}, p_{3,1}\right)\right\} \\
= & \{(0,0),(1,0),(2,0),(3,0),(4,0),(5,0),(6,0.328), \\
& (7,0.552),(8,0.635),(9,0.469),(10,0.141)\}
\end{aligned}
$$

其对应的隶属度集合如下：

$$
p_{3}=\{0,0,0,0,0,0,0.328,0.552,0.635,0.469,0.141\}
$$

其中最大隶属度为 $p_{3, \text { maxi }}=\max \left(p_{3}\right)=0.635$, 它所对应的 宏观异常群严重度 $v_{3, m p}=8$ 。

\section{4. 宏观异常测度空间建立}

宏观异常现象复杂, 宏观异常研究相关数据缺乏, 在这样情况下，要想对某一时空范围出现的宏观异常 群体进行综合度量是不容易的。而对宏观异常的认识 和运用目前主要由一线地震工作者来完成, 由此提出 
一种测度宏观异常群的思路, 即一线地震工作者根据 自身知识和经验将对宏观异常群的综合异常程度的评 判结果进行模糊输入, 对多个评判结果归一化后进行 综合处理, 得到一个共识性的结果。其产生过程如图 4 所示。

\section{1 宏观异常群测度空间的具体建立过程}

我们构建测度空间的过程主要是计算描述宏观异 常群的指标和一线地震工作者评判宏观异常群情景的 综合异常程度结果之间的模糊关系，具体过程如图 5 所示。假设生成了 $\lambda$ 个宏观异常群的模拟情景, 一线
地震工作者对随机出现的宏观异常情景进行评判, 得 到 $k$ 次评价结果, 对宏观异常群的描述指标做降维处 理, 得到描述空间异常群的两个指标: 空间密集度 $c$ 和非空间综合指标 $s$ 。空间密集度可由情景中出现的宏 观异常位置坐标计算出; 非空间综合指标是对宏观异 常群中出现的宏观异常类别、各类别出现次数及单个 宏观异常的异常幅度进行综合得到的，综合的过程用 到了各异常类别的权重, 它的计算来源于 $k$ 次评判结 果。根据得到的样本数据自身构造各自的监控空间, 和评判信息的归一化结果结合进行三维信息扩散, 得 到宏观异常群的测度空间。

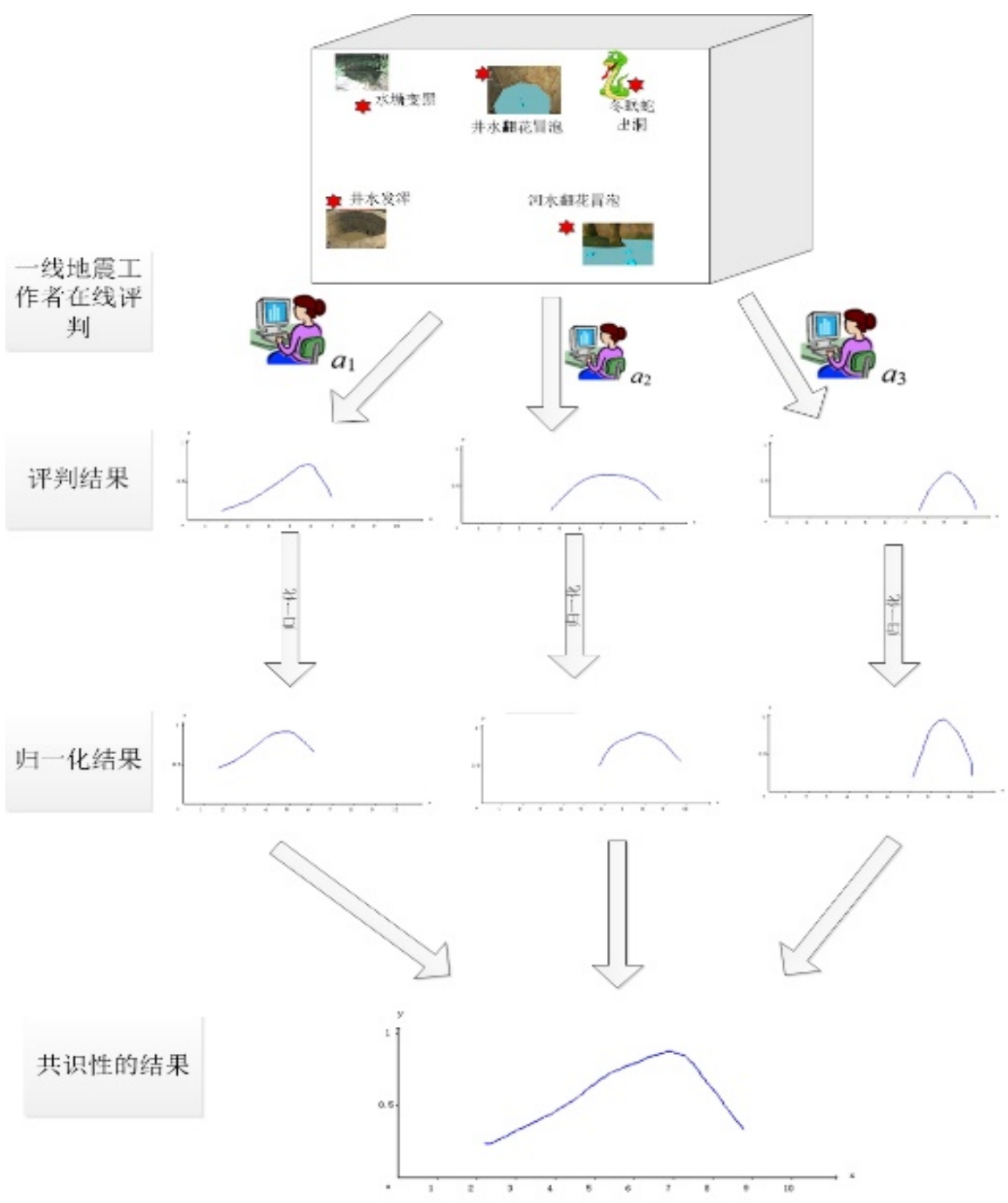

图 4. 一个模拟情景共识性结果的产生过程. 


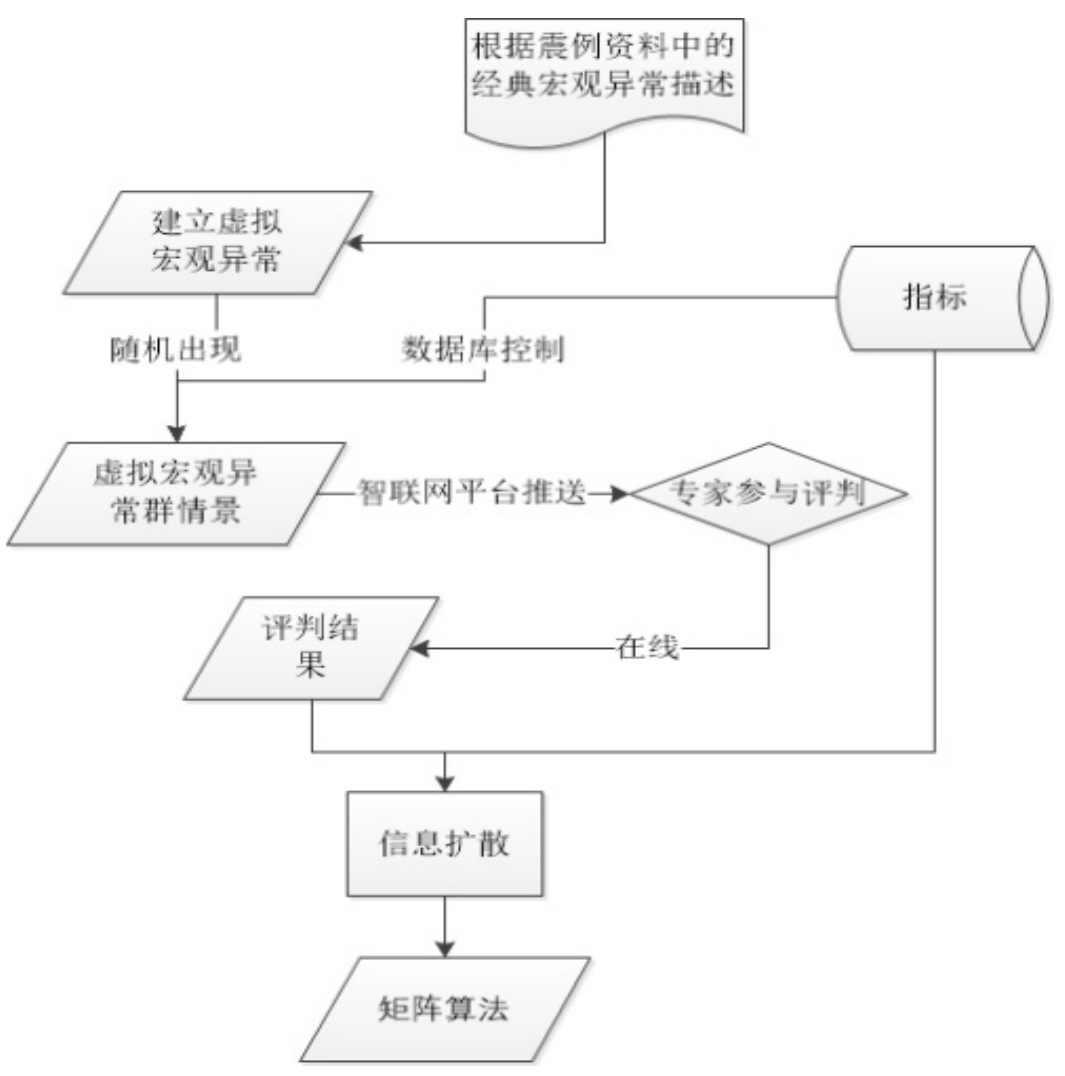

图 5. 宏观异常群测度空间的构建过程

\section{2 宏观异常群的综合指标计算}

为了更好地建立宏观异常的测度空间, 综合度量 宏观异常群, 同时由于样本数量较少, 需要对描述宏 观异常群的变量降维处理, 即把异常种类, 异常起数 和异常幅度综合成一个非空间综合变量作为一个输 入, 空间聚集度作为第二个参量, 把一线地震工作者 的评判结果作为输出, 通过建立三者之间的输入一输 出关键构建宏观异常群的测度空间。首先我们可以从 单个宏观异常信息的形式化表达式里计算出整个宏观 异常群的空间密集度。

\section{3 宏观异常群空间综合指标的建立}

用空间密集度表征宏观异常群的空间属性，指一 定时空范围内出现的宏观异常其在空间上的聚集程 度。本文建立宏观异常群的测度空间时, 其空间综合 指标暂时用单位面积内的宏观异常总量来表示, 即总 起数/面积。 $\left(x_{i j}, y_{i j}\right)$ 表示第 $\mathrm{i}$ 个宏观异常群情景中第 $\mathrm{j}$ 起
异常的位置。据此可计算出空间密集度指标 $c_{i}$ 。对一 个一线地震工作者来说, 他进行某一次评价, 异常情 景是随机出现的。假设进行第 $e$ 次评价时出现了第 $\mathrm{i}$ 个情景。对一个异常情景来说, 它可能出现不止一次。

假设有 $\lambda$ 个虚拟情景被随机推送, 对第 $i$ 个宏观异 常群情景 $S_{i}$ 来说, 设其有 $n$ 起异常, 则所有异常横坐 标和纵坐标集合分别为:

$$
\left\{\begin{array}{l}
x_{i}=\left\{x_{i 1}, x_{i 2}, \cdots x_{i n}\right\} \\
y_{i}=\left\{y_{i 1}, y_{i 2}, \cdots y_{i n}\right\}
\end{array}\right.
$$

其中, $i=1,2, \cdots, \lambda ; j=1,2, \cdots, n$ 。

一定时空范围内宏观异常出现的次数统计即可, 但对于面积的计算有多种方法, 最简单的方法是根据 宏观异常群中异常的位置坐标 $\left(x_{i j}, y_{i j}\right)$ 的最大值最小值 确定的矩形来计算。

本文采用生成最小凸多边形的方法来计算宏观异 常群的面积，其具体的计算过程如下：

进行坐标转换。对宏观异常在 Unity3D 中的世界 
坐标进行坐标转换, 得到转换后的坐标集合为 $\left\{\left(\tilde{x}_{i 1}, \tilde{y}_{i 1}\right),\left(\tilde{x}_{i 2}, \tilde{y}_{i 2}\right), \cdots,\left(\tilde{x}_{i n}, \tilde{y}_{i n}\right)\right\} 。$

生成异常点对应的圆。一个宏观异常对应空间上 一个点, 考虑一个宏观异常辐射周边区域, 对周边区 域有大致的影响范围, 这里假设这个影响范围是以宏 观异常点为圆心, 以一定范围为半径的圆形区域。将 该圆形以离散点的集合 $\left\{\left(\widehat{x}_{i 1}, \hat{y}_{i 1}\right),\left(\widehat{x}_{i 2}, \hat{y}_{i 2}\right), \cdots,\left(\widehat{x}_{i m}, \hat{y}_{i m}\right)\right\}$ 来表示。

生成最小凸多边形。根据离散点的集合 $\left\{\left(\widehat{x}_{i 1}, \hat{y}_{i 1}\right),\left(\widehat{x}_{i 2}, \hat{y}_{i 2}\right), \cdots,\left(\widehat{x}_{i m}, \hat{y}_{i m}\right)\right\}$ 生成最小凸多边形, 它必 须包含所有的点, 同时必须尽可能获得最小的面积。 具体采用的是分而治之算法, 时间复杂度接近于 $\mathrm{O}(\mathrm{n} * \log n)$ ，算法流程如下：

（1）找到对角线。找出点集中左边最上的点, 找到 右边最下的点，可以确定从左上到右下的一个向量 $\vec{a}$ 。

（2）获取对角线左边的顶点序列。将向量 $\vec{a}$ 左边的 距向量最远的点插入序列中, 形成两个新的向量 $\vec{b}_{1}$ 和 $\vec{b}_{2}$ 。获取第一个向量 $\vec{b}_{1}$ 的左边顶点序列, 形成新的两 个向量 $\vec{b}_{3}$ 和 $\vec{b}_{4}$, 如此这般, 直到没有新的顶点序列产 生。接着获取第二个向量 $\vec{b}_{2}$ 的左边顶点序列。直到遍 历所有向量, 不再有左边顶点序列产生。这是一个递 归的过程。

（3）翻转对角线，获得一个右下到左上的向量 $\vec{b}$ 。
（4）获取对角向量 $\vec{b}$ 左边的顶点序列。如同步骤 (2)

一样进行递推, 直到不再产生左边顶点。最后得到的 顶点序列中所有的点就可以构成一个凸多边形，它就 是点集 $\left\{\left(\widehat{x}_{i 1}, \widehat{y}_{i 1}\right),\left(\widehat{x}_{i 2}, \widehat{y}_{i 2}\right), \cdots,\left(\widehat{X}_{i m}, \hat{y}_{i m}\right)\right\}$ 的最小凸多边 形。

计算最小凸多边形的面积 $r$ 。通过前面的处理, 利 用百度地图中开源库的 API 函数计算我们生成顶点序 列的面积, 函数为 BMapLib.GeoUtils.getPolygonArea 得到的就是最小凸多边形的面积。该函数返回 Number 数据类型, 能够计算出多边形面积, 或者由点数组构 建的图形面积, 值得注意的是: 所计算的 polygon 坐标 类型只能是经纬度，且不适合计算自相交多边形的面 积 ${ }^{[7]}$ 。

用最小凸多边形方法确定矩形法计算出的面积如 图 6 所示, 红色气泡表示宏观异常点, 红色虚线表示 最小凸多边形方法的面积范围。

如此就可以计算出宏观异常群的空间密集度, 如 式子(16)所示。

$$
c=\frac{N T}{\gamma}
$$

宏观异常群非空间指标的建立。除了空间因素之 外, 我们把一个宏观异常群情景中出现的种类、数量、 异常幅度等指标进行处理，得到一个非空间的综合指

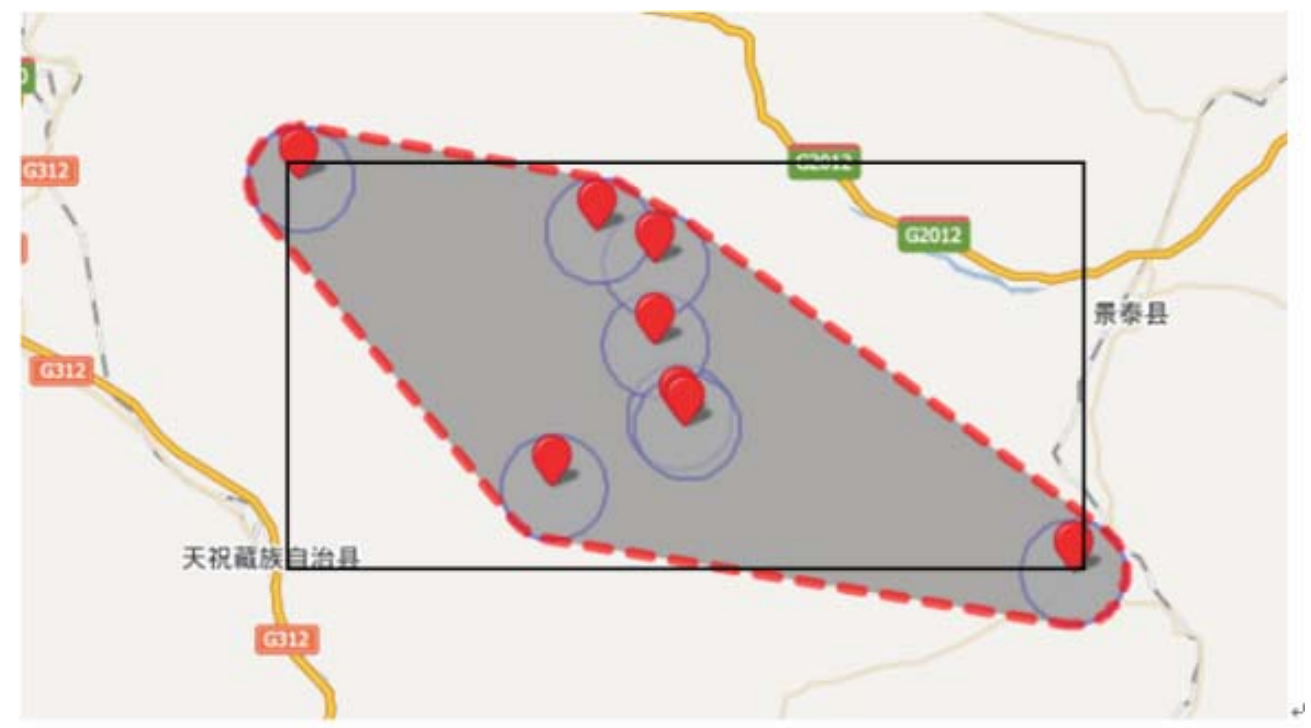

图 6. 最小凸多边形计算宏观异常群面积结果示意 
标 $s$, 其中宏观异常一共分为 5 类。建立该指标的过程 中除了需要情景中出现的宏观异常相关的数据外, 还 需要借助一线地震工作者对相应情景的评判信息。设 第 $e$ 次评价时, 随机出现了第 $i$ 个情景 $S_{i}$ 。共有 $\lambda$ 个情 景, 进行了 $k$ 次评价, 则第 $e$ 次评价对应的 $S_{i}$ 的非空 间信息及其评价信息表示为式 (17):

$$
I_{e}=\left(e, i, N_{i 1}, N_{i 2}, \cdots, N_{i, 5}, v_{e, m p}\right)
$$

其中 $e=1,2, \cdots, k ; i=1,2, \cdots, n$ 。把 $I_{e}$ 作为矩阵的一 行, 共有 $k$ 行。

$I_{e}$ 表示这是第 $e$ 次评价, $e$ 是评价 ID, $i$ 是情景 ID, $N_{i 1}$ 表示第一类异常 $V_{1}$ 在情景 $S_{i}$ 中出现的个数, $v_{e, m p}$ 表 示最大隶属度对应的宏观异常群严重度。

宏观异常群非空间指标的建立主要分两部分进 行。首先进行种类重要性排序, 计算各类别权重值。 对于式（17）的所有类别来说, 若 $N_{i j}>0$ 则令 $N_{i j}=1$ 。 把无差异情景行即每类异常都有的异常情景所在的行 去除, 即若 $N_{i j}>0, j=1,2, \cdots, 5$, 则把第 $i$ 个情景对应 的所有评价信息行去除。再分别针对某类异常进行处 理计算。如对第一类异常 $V_{1}$ 来说, $N_{i 1}=0$, 则把相对 应的第 $i$ 个情景对应的所有评价信息行去除, 然后把 剩下的所有评价 $v_{e, m p}$ 相加并除以剩下的所有评价个 数。最后将所有类别对应的值进行归一化处理。得到 式 (18):

$$
W=\left\{w_{1}, w_{2}, \cdots w_{5}\right\}
$$

第二步把式 (18) 中 $W$ 作为权重值计算综合指标 $S$ 。对第 $i$ 个情景来说,

$$
S_{i}=\sum_{j=1}^{n} w_{V_{i j}} \times a_{i j}, i=1,2, \cdots, m
$$

其中 $w_{V_{i j}}$ 表示第 $i$ 个情景宏观异常 $j$ 的异常类别对应的 权重值, $a_{i j}$ 表示情景 $i$ 中第 $j$ 个宏观异常的异常幅度。

\section{4 建立用模糊关系矩阵表达测度空间的具体算法}

经过前面的预处理，我们已经得到了描述宏观异 常群的两个指标: 非空间指标 $s$ 和空间分布指标 $c$ 。

设有 $n$ 个评价资料，每个资料有三个分量 $s 、 c$ 和 $J$, 记第 $i$ 个评价资料为 $D_{i}=\left(s_{i}, c_{i}, J_{i}\right)$ 。视 $D_{i}$ 为一个信息, $s_{i}, C_{i}, J_{i}$ 为信息分量。其中 $s_{i}, C_{i}$ 对应着某一个宏观异常群 情景。

为了寻求 $s, c, J$ 三个信息分量之间的输入-输出 关系, 得到从 $s, c$ 的离散论域到 $J$ 的离散论域的模糊 关系矩阵 $R$ 的数值，采用三维信息扩散公式对评价资 料 $D$ 构造三维信息矩阵。由于信息分量 $J_{i}$ 的特殊性, 我们不需要再对此分量进行扩散, 只需要对其进行归 一化处理, 就可以将 $J_{i}$ 分配到监控空间中。这样得到 的样本在每个监控点的信息量，可以完整的嵌入到三 维信息扩散公式当中。

（1）分量 $J$ 归一化处理

取宏观异常群严重度离散论域为

$$
v=\left\{v_{1}, v_{2}, \cdots, v_{\beta}\right\}
$$

因为我们的信息分量 $J_{i}$ 比较特殊，它本身就是一 个模糊子集，用扎德记号可表示为

$$
J_{i}=\frac{p_{i 1}}{v_{1}}+\frac{p_{i 2}}{v_{2}}+\cdots+\frac{p_{i \beta}}{v_{\beta}}
$$

可对 $J_{i}$ 进行信息量总和为 1 的归一化处理，可得 $J_{i}$ 在宏观异常群严重度论域上各个分量上的信息分配。

$$
\left\{\begin{array}{l}
q_{i}\left(v_{1}\right)=\frac{p_{i 1}}{p_{i 1}+p_{i 2}+\cdots p_{i \beta}} \\
q_{i}\left(v_{2}\right)=\frac{p_{i 2}}{p_{i 1}+p_{i 2}+\cdots p_{i \beta}} \\
\vdots \\
q_{i}\left(v_{\beta}\right)=\frac{p_{i \beta}}{p_{i 1}+p_{i 2}+\cdots p_{i \beta}}
\end{array}\right.
$$

我们可以选取一个例子进行简单计算, 取 $v=\left\{v_{1}, v_{2}, \cdots, v_{\beta}\right\}=\{0,1,2, \cdots, 10\}$, 第 3 次评价结果为

$$
\begin{aligned}
J_{3}= & \left\{\left(v_{1}, p_{3,1}\right),\left(v_{2}, p_{3,2}\right), \cdots,\left(v_{3,11}, p_{3,11}\right)\right\} \\
= & \{(0,0),(1,0),(2,0),(3,0),(4,0),(5,0),(6,0.328), \\
& (7,0.552),(8,0.635),(9,0.469),(10,0.141)\}
\end{aligned}
$$

它是一个模糊子集，用扎德记号可表示为 


$$
\begin{aligned}
J_{3}=\frac{p_{i 1}}{v_{1}}+\frac{p_{i 2}}{v_{2}}+\cdots+\frac{p_{i \beta}}{v_{\beta}} \\
=\frac{p_{31}}{v_{1}}+\frac{p_{32}}{v_{2}}+\cdots+\frac{p_{3,11}}{v_{11}} \\
=\frac{0}{0}+\frac{0}{1}+\frac{0}{2}+\frac{0}{3}+\frac{0}{4}+\frac{0}{5}+\frac{0.328}{6}+\frac{0.552}{7}+ \\
\frac{0.635}{8}+\frac{0.469}{9}+\frac{0.141}{10}
\end{aligned}
$$

隶属度为 0 时可以不表示, 则

$$
J_{3}=\frac{0.328}{6}+\frac{0.552}{7}+\frac{0.635}{8}+\frac{0.469}{9}+\frac{0.141}{10}
$$

根据式(2-50)对其进行归一化处理:

$$
\left\{\begin{array}{l}
q_{3}\left(v_{1}\right)=\frac{0}{0.328+0.552+0.635+0.469+0.141}=0 \\
q_{3}\left(v_{2}\right)=\frac{0}{0.328+0.552+0.635+0.469+0.141}=0 \\
\vdots \\
q_{3}\left(v_{11}\right)=\frac{0.141}{0.328+0.552+0.635+0.469+0.141}=0.066
\end{array}\right.
$$

（2）构造监控空间

对于 $s_{i}$ 和 $c_{i}$ 两个指标, 可以用常规信息扩散方法 来处理。首先构造关于 $s$ 和 $c$ 的监控点序列。设非空 间指标 $s$ 的监控点序列为

$$
U=\left\{u_{1}, u_{2}, \cdots, u_{k}\right\}
$$

设空间指标 $c$ 的监控空间为

$$
O=\left\{o_{1}, o_{2}, \cdots o_{r}\right\},
$$

以宏观异常群的非空间指标 $s$ 为例, 来说明如何 构造相应的离散论域作为监控空间。监控空间通常数 据本身息息相关，具体构造过程如式（23）所示。

$$
\left\{\begin{array}{l}
s=\left\{s_{1}, s_{2}, \cdots, s_{n}\right\} \\
U=\left\{u_{1}, u_{2}, \cdots, u_{\rho}\right\} \\
u_{1}=\min (s)-\Delta u / 2 \\
u_{i+1}=u_{i}+\Delta u \\
\rho=(\max (s)-\min (s)) /(\Delta u)+1 \\
0<\Delta u \leq \min _{s_{i} \neq s_{j}}\left(\left|s_{i}-s_{j}\right|\right) \\
i, j=1,2, \cdots, n
\end{array}\right.
$$

式 (23) 中, $s$ 表示原始样本数据, $U$ 表示监控点 序列, $u_{i}$ 表示序列 $U$ 中的第 $i$ 个监控点, $\rho$ 表示 $U$ 中 需要构造的监控点个数, $\Delta u$ 表示监控点序列的步长。
同理可构造宏观异常群的空间密集度 $c$ 的监控空间 $O$ 。

(3) 计算扩散系数和信息矩阵

三维正态信息扩散估计函数如式 (24) 所示 ${ }^{[8]}$ :

$$
\mu_{u_{k} o_{j}}=\exp \left[-\frac{\left(u_{k}-s_{i}\right)^{2}}{2 h_{s}^{2}}\right] \exp \left[-\frac{\left(o_{j}-c_{i}\right)^{2}}{2 h_{c}^{2}}\right] \exp \left[-\frac{\left(v_{j}-z_{i}\right)^{2}}{2 h_{z}^{2}}\right]
$$

其中, $\left(s_{i}, c_{i}, z_{i}\right)$ 是样本点, $u_{k}, o_{j}, v_{l}$ 是对应的监控点, $h_{s}, h_{c}, h_{z}$ 是扩散系数。

将式（22）和式（24）结合，进行改造得到适合 本研究的三维信息扩散函数如式（25）所示:

$$
\mu_{u_{k} o_{j} v_{l}}\left(s_{i}, c_{i}, E_{i}\right)=\exp \left[-\frac{\left(u_{k}-s_{i}\right)^{2}}{2 h_{s}^{2}}\right] \exp \left[-\frac{\left(o_{j}-c_{i}\right)^{2}}{2 h_{c}^{2}}\right] q_{i}\left(v_{l}\right)
$$

其中, 扩散系数 $h_{s}, h_{c}$ 可以根据式 (26) 计算得到,

$$
h=\left\{\begin{array}{l}
0.8146(b-a), n=5 \\
0.5690(b-a), n=6 \\
0.4560(b-a), n=7 \\
0.3860(b-a), n=8 \\
0.3362(b-a), n=9 \\
0.2986(b-a), n=10 \\
2.6851(b-a) /(n-1), n \geq 11
\end{array}\right.
$$

其中, $b=\max _{1 \leq i \leq n}\left(x_{i}\right), \quad a=\min _{1 \leq i \leq n}\left(x_{i}\right)$ 。

$\mu_{u_{k} o_{j} g_{l}}\left(s_{i}, C_{i}, J_{i}\right)$ 可以简写为 $\mu_{k j l}$, 接下来要将各样 本对 $\left(u_{k}, o_{j}, v_{l}\right)$ 的信息扩散量进行叠加, 以 $Q_{k j l}$ 表示,

$$
Q_{k j l}=\sum_{i=1}^{n} \mu_{u_{k} o_{j} \nu_{l}}\left(s_{i}, c_{i}, J_{i}\right)
$$

为了更清晰地表达三维信息矩阵，我们对其做一 下简单变换处理, 用 $Q_{k l}^{(j)}$ 表示 $Q_{k j l}$ 。

\section{(4) 计算模糊关系矩阵}

将原始信息矩阵用式 (28) 和式 (29) 进行处理, 可得到空间指标 $c$ (空间密集度) 和宏观异常群自身的 非空间指标 $s$ 与宏观异常严重度的模糊关系矩阵 $R=\left\{r_{k l}^{(j)}\right\}$ 。这个模糊关系矩阵就是得到的测度空间。

$$
\begin{gathered}
H_{l}=\max _{\substack{1 \leq j \leq \leq \\
1 \leq k \leq K}}\left\{Q_{k l}^{(j)}\right\} \\
r_{k l}^{(j)}=Q_{k l}^{(j)} / H_{l}, \quad j=1,2, \cdots ; k=1,2, \cdots
\end{gathered}
$$

\section{5. 结论和讨论}

本文主要基于宏观异常研究进展缓慢, 缺乏测度 空间的支撑, 很难开展系统性科学研究的现状, 提出 
了宏观异常群的概念, 研究了如何描述和表达宏观异 常群信息，怎样建立描述宏观异常群的空间综合指标 ——空间密集度和非空间综合指标，如何基于此构建 宏观异常群的测度空间，那就是研制智联网平台，邀 请多位一线地震工作者在线对模拟的宏观异常情景进 行评判, 将评判结果作为输出, 宏观异常群的空间综 合指标和非空间综合指标作为输入，利用三维信息扩 散技术形成共识性的地震宏观异常测度空间, 为实现 地震宏观异常的综合量化提供理论基础。同时，将模 糊量化输入和综合处理的理念引入, 为智联网的信息 沟通提供了一个新的手段。

宏观异常种类繁多, 异常形态复杂多样。异常形 态的复杂性和多样性是异常形成条件和异常机理复 杂、多样性的反应。例如不同井水位的异常幅度差别 很大, 大者可达几十厘米, 甚至 1 米以上, 小者仅几 毫米。从统一层面上对宏观异常的异常幅度分级划分 是很困难的, 但是异常幅度本身也存在一定信息量, 本研究为了将单个异常的异常幅度作为描述宏观异常 的一个属性, 从而为描述宏观异常群贡献信息, 将其 划分为两个级别。划分方法虽然稍显粗粘, 也是我们 度量宏观异常群的综合异常程度的一个必不可少的指 标, 在实际的宏观异常核查过程中, 专家或者一线地 震工作者可以根据具体异常点过往表现情况, 结合自 身经验知识对单个异常的强烈明显程度进行判断。

\section{参考文献}

[1]C. F. Huang. Internet of intelligences in risk analysis for online services, Journal of Risk Analysis and Crisis Response,1(2):110-117,2011.

[2]车用太,鱼金子,刘成龙.地震宏观异常与临震预测成功的 希望[J].国际地震动态,2012(6):138-138.

[3]许敦煌.汶川大地震前宏观异常的现场调查 [J]. 地 震, 2010,30(2):121-133.

[4]张小涛,张永仙,许敦煌.汶川 8.0 级地震前后宏观异常现象 分析 [J].地震, 2009,29(2):104-117.

[5]王蔚丹,黄崇福.建立共识性地震宏观异常测度空间的研究. 大数据时代的风险分析和危机反应--中国灾害防御协会 风险分析专业委员会第六届年会论文集 [C].中国,长 沙,2016.11.4-6:908-913.

[6]李坤,李秀英.地下水宏观异常与强震临震预报 [J]. 华北地 震科学, 1997,15(4):57-63.

[7]http://api.map.baidu.com/library/GeoUtils/1.2/docs/symbols/ BMapLib.GeoUtils.html.

[8]黄崇福.自然灾害动态风险分析的一个虚拟案例 $[\mathrm{J}]$. 灾害 学,2015(4):1-11. 